\title{
Limits of BOTDA Range Extension Techniques
}

\author{
Xabier Angulo-Vinuesa, Alejandro Dominguez-Lopez, Alexia Lopez-Gil, Juan D. Ania-Castañon, \\ Sonia Martin-Lopez and Miguel Gonzalez-Herraez
}

\begin{abstract}
Brillouin-based temperature and strain sensors have attracted great attention of both the academic and industrial sectors in the past few decades due to their ability to perform distributed measurements. Particularly, Brillouin Optical Time Domain Analysis (BOTDA) systems have been applied in many different scenarios, proving particularly useful in those requiring especially wide coverage ranging extremely long distances, such as in civil structure monitoring, energy transportation or environmental applications. The extension of the measuring range in these sensors has therefore become one of the main areas of research and development around BOTDA. To do so, it is necessary to increase the Signal to Noise Ratio (SNR) of the retrieved signal. So far, several techniques have been applied in order to achieve this goal, such as pre-amplification before detection, pulse coding or Raman amplification. Here, we analyze these techniques in terms of their performance limits and provide guidelines that can assist in finding out which is the best configuration to break current range limitations. Our analysis is based on physical arguments as well as current literature results.
\end{abstract}

Index Terms-Brillouin scattering, distributed optic fiber sensing, distributed Raman scattering, optical fibers, optical pulse coding.

\section{INTRODUCTION}

A MONG the different available techniques to perform distributed measurements of strain and temperature, Brillouin Optical Time-Domain Analysis (BOTDA) [1,2] is presently recognized as one of the most consolidated. This fiber sensing technology is widely used in different application domains (civil engineering, pipelines, fire detection, etc.).

Manuscript received February 1, 2015; revised April 14, 2015; accepted -------- --, 2015. Date of current version --------- --, 2015. This work was supported by the European Research Council through Starting Grant U-FINE (Grant no. 307441), the Spanish "Plan Nacional de I+D+i" through projects TEC2012-37958-C02-01, TEC2012-37958-C02-02 and TEC2013-45265-R, the Spanish "Ministerio de Ciencia e Innovación" through project RAMAS TEC2011-27314, the INTERREG SUDOE program ECOAL-MGT, the European Comission though ITN ICONE CA-608099 and in part by the Comunidad de Madrid under projects EDISON (CCG2014/EXP-072) and SINFOTON-CM:S2013/MIT-2790. The work of S. Martin-Lopez was supported by the Spanish "Ministerio de Ciencia e Innovación" through a "Ramón y Cajal" contract.

X. Angulo-Vinuesa, A. Dominguez-Lopez, A. Lopez-Gil, S. Martin-Lopez and M. Gonzalez-Herraez are with the Departamento de Electrónica, Universidad de Alcalá, Edificio Politécnico, Alcalá de Henares 28871, Spain (e-mail: xabier.angulo@uah.es; alejandro.dominguezl@uah.es, alexia.lopez@uah.es; sonia.martinlo@uah.es,miguelg@depeca.uah.es).

J. D. Ania-Castañon is with the Instituto de Óptica, Consejo Superior de Investigaciones Científicas, Serrano 121, Madrid 28006, Spain (e-mail: jd.ania@csic.es).
BOTDA technology finds its root on the non-linear optical effect occurring in optical fibers and called Stimulated Brillouin Scattering (SBS) [3]. SBS is an acousto-optic process that manifests as a narrowband amplification of a probe beam when an intense coherent pump light beam is introduced through the opposite end of a single-mode fiber. The distributed feature is provided to the BOTDA by pulsing the pump wave and analyzing the retrieved probe wave as function of the time-of-flight of the pump pulse within the fiber, as depicted in Fig.1.

Range and resolution are two of the most important features of these systems, which are normally limited to $20-30 \mathrm{~km}$ with 1-2 meter resolution in standard systems [4]. Attenuation, an intrinsic fiber feature, is the limiting factor in terms of range as it reduces the intensity of the signals within the fiber, therefore decreasing the Signal to Noise Ratio (SNR) as the monitored distance increases. Resolution, which is set by the spatial length of the employed pump pulses, is restricted by the associated issues when the pulse duration is reduced. First, a spectral broadening will manifest on the retrieved wave (leading to a higher uncertainty) and also a shorter interaction length will be obtained among the pump and probe wave, leading to a corresponding SNR decrease. Both factors lead to a non-proportional increase of the measurement uncertainty when the pump pulse is scaled in the sub-meter range. An appropriate balance among range and resolution should be found to properly fulfill the required system specifications for each application.

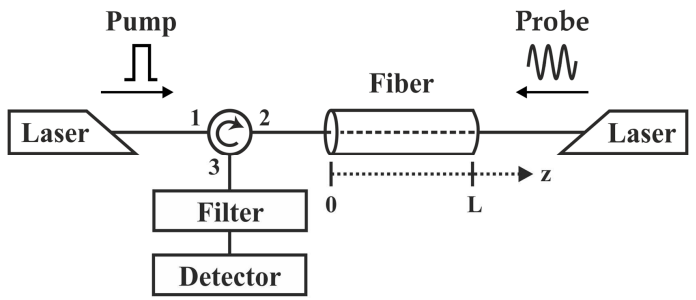

Fig. 1. Schematic depiction of a Brillouin Optical Time Domain Analysis (BOTDA) system.

Recent times have seen a gradual increase in the number of large infrastructures that require intensive monitoring. The size of these structures makes its health safety monitoring extremely critical, as well as financially very demanding with conventional sensing schemes, especially for constructions crossing vast unmanned areas (deep subsea ducts, pipelines crossing desserts, umbilical cables for offshore wind-farms, etc.). Thus, new approaches are needed to extend the range of conventional distributed sensors for these infrastructures [5]. 
In this paper, we analyze the different available techniques for range enhancement in BOTDA sensors, and their effect in the Figure of Merit (FoM) of the BOTDA in terms of sensing distance. First, we describe the range limitations of standard BOTDA systems; then we provide a detailed description of the available techniques in order to extend the distance of the Brillouin sensors where an extensive discussion of the expectable limits of each technique is performed. Finally, the effect of the combination among techniques is described to conclude which is the path to follow when enhancing the sensing distance of such systems.

\section{CONVENTIONAL BOTDA RANGE LIMITATIONS}

As mentioned in the Introduction section, in BOTDA systems, a pulsed pump wave interacts with a counterpropagating continuous probe wave through SBS. From that interaction, the power gained by the probe wave (from the pump wave), as represented in Fig.2, can be expressed as [6]:

$$
\Delta P_{S}(z)=\frac{g_{B}}{A_{e f f}} P_{P}(z) P_{S}(z) \Delta z
$$

where $\Delta P_{S}$ is the local power transfer to the probe wave, $g_{B}$ is the Brillouin gain coefficient, $A_{\text {eff }}$ is the nonlinear effective area, $P_{P}$ and $P_{S}$ are the pump and probe powers respectively and $\Delta z$ is the spatial resolution. Assuming negligible pump depletion [7], the pump wave will be only affected by linear fiber attenuation $(\alpha)$ :

$$
\Delta P_{S}(z)=\frac{g_{B}}{A_{e f f}} P_{P i} P_{S i} e^{-\alpha L} \Delta z
$$

where $P_{P i}$ and $P_{S i}$ are the input pump and probe powers. In BOTDA systems, the amplified probe wave is detected at the $z=0$ position (see Fig.1), so the retrieved signal at that position, in the worst case situation (in the far end of the fiber), will suffer from linear attenuation once again:

$$
\Delta P_{S}^{0}=\frac{g_{B}}{A_{\text {eff }}} P_{P i} P_{S i} e^{-2 \alpha L} \Delta z
$$

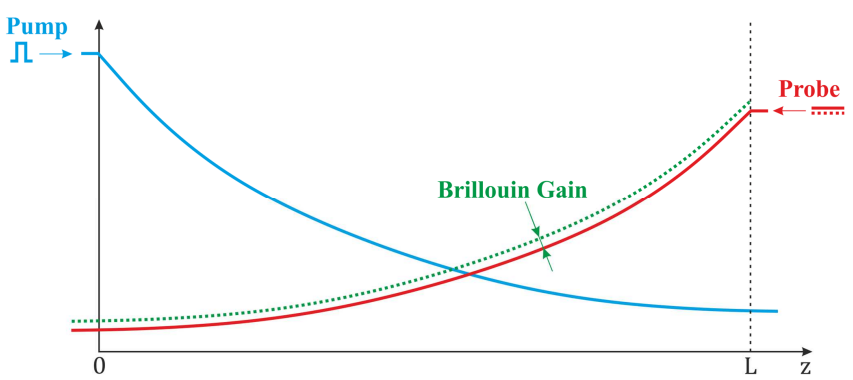

Fig. 2. Graphical representation of the Brillouin interaction among a pulsed pump wave and a continuous probe wave.

Note that, according to Eq. 3, the effect of fiber attenuation is reflected in a squared exponential decay with distance. Based on this equation, we can conclude that in terms of signal amplitude, a resolution increase by a factor of 10 , which is considered a breakthrough in the field, is just equivalent to raising the sensing range by only $25 \mathrm{~km}$, which cannot be considered as a substantial improvement when dealing with long-range systems.

Another issue regarding range limitations lays on the fact that on BOTDA systems two waves are employed that access the fiber from both ends. If it is considered that the signals emerge from a unique sensing unit, for linear sensing applications (i.e. pipelines, umbilical cables, etc.) the detected amplified probe wave has to travel up to the position where the measurement is developed and back. Consequently, if no active elements are present along the sensing fiber, the linear sensing range is limited to half of the nominal range of the instrument.

In terms of signal power levels, the probe wave is limited by different reasons depending on the exact configuration used. When using a Single Sideband Probe (SSP) scheme, the restriction arises from the SBS depletion of the pump wave $[7,8]$. For long-range systems (much longer than the typical effective length in fibers, $1 / \alpha \approx 22 \mathrm{~km}$ ), the probe power limit can be estimated to be $\sim 40 \mu \mathrm{W}$ [7]. When dealing with a Double Sideband Probe (DSP) configuration, the SBS threshold sets the limit at $\sim 5 \mathrm{~mW}$ in the same conditions [7], which is two orders of magnitude larger. On the opposite side, the pump wave power will be restricted by the onset of Modulation Instability (MI) [9], which sets the maximum power at $100 \mathrm{~mW}$ approximately in these conditions. If we now introduce these figures in $\mathrm{Eq} \mathrm{3}$, it turns out that, over a $100 \mathrm{~km}$ sensing scheme, these factors only allow $\mathrm{nW}$ power variations that should be measured over typically $100 \mathrm{MHz}$ bandwidth detectors (for meter scale resolutions). Considering typical noise powers in detection $(2.5 \mathrm{pW} / \sqrt{\mathrm{Hz}})$, this implies that at least several thousands of averages will be required in conventional BOTDA configurations, with the consequent measuring time increase.

The power limitations imposed by SBS pump depletion can be overcome by reducing the interaction time among the pump and probe waves. Nonlocal effects arise from the cumulative energy exchange between the pump and probe waves. Thus a reduction in the interaction length among these signals leads to a reduction in the SBS-induced depletion (and hence the errors). Two techniques have been developed in order to avoid such issue and they are based on Time and Frequency Division Multiplexing (TDM and FDM respectively). TDM was first proposed by Zornoza et al. [10] and is based on pulsing the probe wave so that the interaction length among pump and probe is reduced [11]. This technique allows using a higher probe power and smaller number of averages, however the measuring time is usually increased. The FDM technique, developed by Dong et al. in [12] bases its principle on employing different fibers with different Brillouin Frequency Shifts (BFS) thus the effective Brillouin interaction is restricted to one resonant Brillouin frequency for each section instead of just one frequency for the whole fiber. The overall effect is similar to the previous configuration (a reduction in the SBS interaction length among pump and probe), however the situation demands extended measuring frequency spans and also may be impractical in existing installations where the fibers cannot be arbitrarily chosen. 
Both techniques show some drawbacks, such as an added setup complexity and an increased measuring time for the TDM and poor scalability and the requirement of using different fibers for the FDM. In any case, they have already been tested as BOTDA range increasing techniques achieving $100 \mathrm{~km} \mathrm{[13]} \mathrm{and} 150 \mathrm{~km} \mathrm{[12]} \mathrm{with} 2$ meter resolution for the TDM and FDM procedures respectively. These values have been obtained through the application of resolution increase techniques, such as the Differential Pulse-width Pair (DPP) [14]. The DPP technique bases its performance on obtaining independent BOTDA traces with long pump pulses with different widths. These traces are then subtracted, being the difference among pulses the resultant resolution. Thus, through this technique somewhat longer distances can be obtained as the employed pump pulses are longer than the resultant resolution. Besides, the FDM demonstration utilized in-line EDFAs within the fiber. Due to these technical features we have decided not to include the TDM and FDM techniques as a part of our analysis, although their effectiveness is well known and satisfactorily tested as a range extension technique.

As we can see, range increase in BOTDA systems is not a trivial matter considering all the aforementioned limitations. Thus, in the following sections, a description of the present available techniques that can assist on increasing measuring distance will be presented.

\section{LIMITS OF BOTDA RANGE EXTENSION SOLUTIONS}

As stated, in this section a description of the available techniques to overcome the aforementioned problems that limit measurement range will be developed and the associated performance issues will be discussed. The first technique is based on employing a pre-amplification stage before detection. This methodology mainly faces the problem of the gain limit that can be reached before having too much SNR degradation. The second procedure bases its principle on coding the pulsed pump signal with a known bit sequence to increase the SNR of the retrieved signal using algebraic operations. Obviously, the longer the code the better the SNR improvement, although affordable code lengths cannot enhance the quality as much as desired in some cases. Finally, the use of distributed Raman amplification is analyzed. This technique transforms the sensing fiber into an amplifying element at the expense of introducing Amplified Spontaneous Emission (ASE) noise and transferring a large amount of Relative Intensity Noise (RIN) from the Raman pumps to the measured signal. The novelty of this discussion is that the expectable improvement in terms of SNR in each case is quantified, providing a measure of the expectable limits of each technique.

\section{A. Pre-Amplification}

A simple way of improving the SNR of the retrieved signal is based on using a pre-amplifier system just before detection. Usually, this stage is formed by introducing an Erbium Doped Fiber Amplifier (EDFA), which may provide up to $30 \mathrm{~dB}$ of gain.
When dealing with elements that provide Gain $(G)$, it is important that all possible noise sources are taken into account. At the photodetector level, it is important to consider the thermal and shot noises [15]:

$$
\begin{aligned}
& \left\langle i_{\text {ther }}^{2}\right\rangle=4 k T \frac{B_{e q}}{R_{f}}+3.7 k T \pi^{2} \frac{C_{t}^{2} B_{e q}{ }^{3}}{g_{m}} \\
& \left\langle i_{\text {shot }}^{2}\right\rangle=2 e\left(\frac{\eta e G\left(P_{s}+P_{c}\right)}{h v}+I_{d}\right) B_{e q}
\end{aligned}
$$

where $k$ is the Boltzman constant $\left(1.38 \cdot 10^{-23} \mathrm{~J} / \mathrm{K}\right)$, T is the temperature $(296 \mathrm{~K}), B_{e q}$ is the equivalent noise bandwidth that is related to the electrical bandwidth of the detector $\left(B_{e}=100 \mathrm{MHz}\right)$ as $B_{e q}=\frac{\pi}{2} B_{e}, R_{f}$ is the transimpedance gain resistance $(40 \mathrm{~K} \Omega), C_{t}$ is the total input capacitance of the detector (typ. $8 \cdot 10^{-12} \mathrm{~F}$ ), $g_{m}$ is the FET transconductance (typ. $\left.5 \cdot 10^{-3} \mathrm{~S}\right), e$ is the electronic charge $\left(1.6 \cdot 10^{-19} \mathrm{C}\right), \eta$ is the quantum efficiency of the detector (typ. 0.63 for InGaAs detectors of this kind), $G$ is the EDFA gain, $P_{S}$ and $P_{c}$ are the amplified signal amplitude and the DC component respectively $\left(5 \cdot 10^{-9}\right.$ and $\left.5 \cdot 10^{-6} \mathrm{~W}\right)$ calculated considering a dual sideband scheme with an input probe power of $500 \mu \mathrm{W}$ and a $100 \mathrm{~km}$ sensing range, $h$ is the Planck constant $\left(6.63 \cdot 10^{-}\right.$ $\left.{ }^{34} \mathrm{~J} \cdot \mathrm{s}\right), v$ is the operating frequency $\left(193.55 \cdot 10^{12} \mathrm{~Hz}\right)$ and $I_{d}$ is the dark current of the detector (typ. $0.15 \cdot 10^{-9} \mathrm{~A}$ ).

As can be observed, thermal noise scales with the temperature and the electrical gain in the photodetector $\left\langle i_{\text {ther }}^{2}\right\rangle \propto k T / R$. Shot noise arises because of the discrete nature of the photodetection process, and turns out to be proportional to the light power, hence $\left\langle i_{\text {shot }}^{2}\right\rangle \propto G$. Besides photodetector noises, EDFA amplifiers also introduce a large amount of noise. EDFAs produce broadband noise all over the amplification bandwidth known as ASE noise [16]. This is caused by the de-excitation process of the previously excited erbium electrons that produce the amplification. When this deexcitation phenomenon arises spontaneously, a photon is generated that has no coherence with the incoming optical signal. This harmful noise source produces a beating in the photodetector with the amplified signal and with itself, while introducing some additional shot noise (which is usually small) [15]:

$$
\begin{gathered}
\left\langle i_{\text {sig-ASE }}^{2}\right\rangle=\frac{2(\eta e)^{2}}{h v} F G^{2}\left(P_{s}+P_{c}\right) B_{e q} \\
\left\langle i_{A S E-A S E}^{2}\right\rangle=(\eta e)^{2} F^{2} G^{2} B_{o} B_{e q} \\
\left\langle i_{A S E-s h o t}^{2}\right\rangle=2 e^{2} \eta G F B_{o} B_{e q}
\end{gathered}
$$

where $F$ is the optical noise figure of the EDFA, given by $\frac{1+2 n_{s p}(G-1)}{G}$ and being $n_{s p}$ the spontaneous emission factor (ideally 1$)$, and $B_{o}$ is the optical bandwidth $\left(50 \cdot 10^{9} \mathrm{~Hz}\right)$.

The sig-ASE and ASE-ASE terms are proportional to the square of the gain hence: $\left\langle i_{\text {sig-ASE }}^{2}\right\rangle \propto G^{2}$ and $\left\langle i_{A S E-A S E}^{2}\right\rangle \propto G^{2}$. Also, the ASE-ASE and the ASE-shot terms depend on the optical bandwidth of the signal entering the detector.

The optical SNR can be evaluated in terms of the currents of 
the detected signal and can be expressed as [15]:

$$
S N R_{o}[d B]=10 \log \left[\frac{G P_{s} \frac{\eta e}{h v} \sqrt{N}}{\sqrt{\sum\left\langle i^{2}{ }_{\text {noise }}\right\rangle}}\right]
$$

where $N$ is the number of averages performed on the retrieved signal and $\left\langle i^{2}{ }_{n o i s e}\right\rangle$ is the current of the different noise sources.

Based on the proportionalities of each noise source, it can be easily seen that the amplification process is only interesting to overcome the thermal noise contribution in the detector. Beyond this limit, the other noise terms grow at a rate proportional to the gain $(G)$ or gain squared $\left(G^{2}\right)$, therefore the SNR is in all cases kept or even reduced.

In Fig.3a, we present a graphical representation of the variation of the different noise sources as a function of the gain in the amplifier. As we can see, thermal noise is dominant for gain values below $7.5 \mathrm{~dB}$. At this point, the onset of sigASE noise starts to dominate, with its well-known quadratic gain dependence. It is important to note that, in this case, the optical filtering has little impact in terms of the SNR (still, it must be stressed that the Rayleigh contribution from the pump pulse is considered eliminated by the filter, which imposes a strong roll-off in the filter edge between pump and probe). In some other cases with lower probe input power, the dominant contribution might be given by ASE-ASE noise. In the latter case, sharper optical filters would allow shifting the optimum gain to higher values and achieving further SNR improvement, however the system would be in most cases much more complex from the point of view of thermal design. As it can be seen in Fig 3(b), the optimum gain in terms of SNR appears at roughly $12 \mathrm{~dB}$, giving a SNR improvement of $7.5 \mathrm{~dB}$ (optical). According to Eq. 3, this SNR improvement is equivalent to a range extension of $\sim 19 \mathrm{~km}$. While this is important, it is not enough in many applications, especially those targeting sensing ranges beyond $100 \mathrm{~km}$.

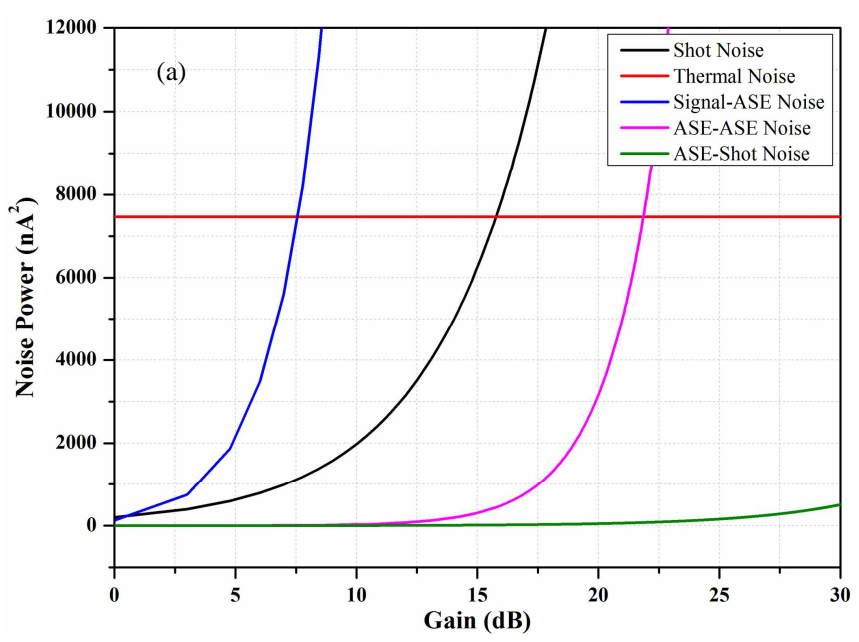

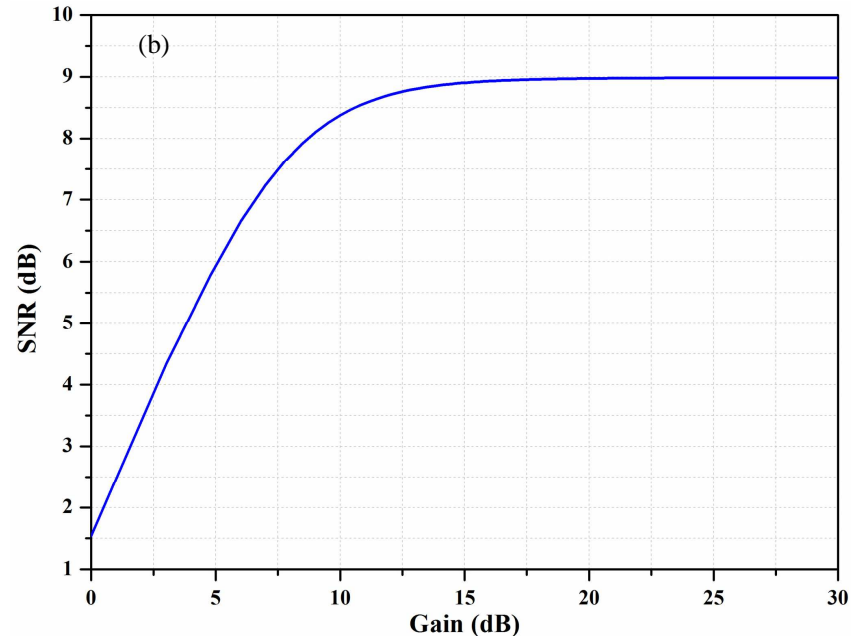

Fig. 3. (a) Noise power in detection as a function of the preamplifier gain, calculated in the conditions of the text; (b) SNR evolution in the same conditions. For this case, 1024 averages have been considered.

\section{B. Pulse Coding}

This SNR enhancement technique [17], extensively developed on BOTDA systems over the last years (see, e.g. $[18,19])$, bases its principle on coding the pump wave with a known pulse sequence. The simplest form of coding that has been widely demonstrated is the simplex coding. When introducing a coded sequence of pulses within the fiber, the amplified probe wave is recovered as a linear superposition of all the traces produced by each independent pulse. This phenomenon is schematically represented in Fig.4.
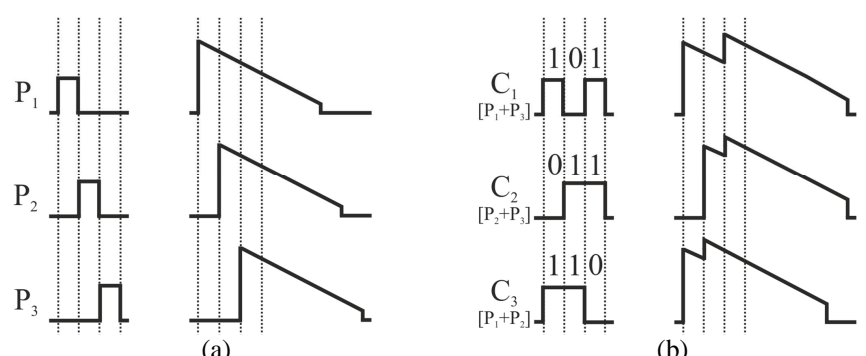

(b)

Fig. 4. Illustration of the retrieved signal with a single pulse (a) and with a linear combination of pulses (b).

Obviously, the fact that superposed traces are present within the fiber increases the received energy level, thus increasing the SNR. After the signal retrieval, through simple algebraic operations, it is possible to isolate each of the measured traces to obtain the equivalent of a single pulse trace. The gain in signal to noise ratio produced by the code can be expressed as:

$$
g_{\text {cod }}=\frac{L_{C}+1}{2 \sqrt{L_{C}}}
$$

where $g_{c o d}$ is the code gain (equivalent to the overall SNR improvement in the trace retrieval) and $L_{C}$ is the code length.

As it is visible, the obtained expression scales with the square root of the code-length $\left(\sqrt{L_{C}}\right)$, accordingly for longer code lengths, more energy will be measured with the consequent SNR improvement. Nonetheless, it has to be taken 
into account some practical limitations to extend the range indefinitely: first, the whole principle of the coding method relies on ensuring the linearity of the detection stage in the conditions of the measurement. The linearity has to be good enough to ensure that a 1 bit change in the code is reliably measured. Therefore, the non-linearity of the detector at the probe power level has to be better than $1 / L_{C}$. According to linearity measurements in conventional InGaAs photodiodes [20], this starts to be extremely challenging for $L_{C}>1000$. In addition, nonlinear distortion in fibers or intermediate active and passive elements is also a concern, as they destroy the linearity assumption. A second main limitation is based on the own nature of the employed pulse format, e.g. Non-Return-toZero (NRZ) or Return-to-Zero (RZ) pulses. In the work presented by Soto et al. in [21], it is clearly described the limitations of the pulse coding method in BOTDA systems, in particular concerning the use of RZ and NRZ pulses. In the NRZ case, the employed pulse format does not allow to properly de-excite the acoustic wave, which can be detrimental for the decoding process considering the desired linear response of the fiber to the different pump codewords. This issue can degrade the SNR of the retrieved signal in more than $5 \mathrm{~dB}$ for $1 \mathrm{~m}$ spatial resolutions [22]. The use of low duty cycle RZ pulses alleviates the problem, but raises other issues in terms of implementation. An optimization of the used pulse format is therefore compulsory in each case, thereby reducing flexibility. Lastly, signal processing time also has to be taken into consideration, although it should not be a big issue in efficient implementations. Considering all these reasons, we can say that, for affordable pulse code lengths ( 511 bits $)$, the SNR enhancement is approximately $10 \mathrm{~dB}$, which implies a range increase of roughly $25 \mathrm{~km}$.

\section{Raman Amplification}

The last technique that has been successful in extending the range of BOTDA sensors is based on introducing an artificial transparency in the sensing fiber by using distributed Raman amplification. A small, fully distributed gain along the sensing fiber is created through stimulated Raman scattering [23]. The distributed gain is engineered to partially or totally compensate the intrinsic fiber loss $(\alpha=0.2 \mathrm{~dB} / \mathrm{km})$ and thus maintain the pump and probe power levels that secure a sufficient signal all along the sensing fiber. In essence, it is a way of achieving a more homogeneous interaction all along the sensing fiber. This effect then, reduces the limitation of the double attenuation suffered by the detected probe wave, and allows a sensing range increase.

Stimulated Raman gain [23] is created in the fiber by pumping the fiber at a shorter wavelength than the signal. Raman scattering produces a broadband $(\sim 7 \mathrm{THz})$ gain curve that is downshifted by $\sim 13 \mathrm{THz}$ with respect to the pumping source. Thus, for achieving efficient gain at $1550 \mathrm{~nm}$, it is typically required to introduce a pump some $100 \mathrm{~nm}$ below (around $1450 \mathrm{~nm}$ ). This is normally done by using Wavelength Division Multiplexers (WDM) able to couple the BOTDA signals and the Raman pump, as represented in Fig.5.

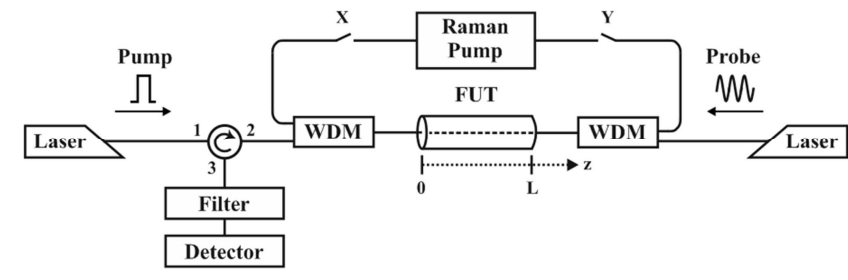

Fig. 5. General configuration of a BOTDA assisted by Raman amplification.

So far, several studies have proven the efficiency of this technique in order to compensate fiber losses and increase the sensing range. Different configurations can be incorporated to the standard BOTDA scheme, such as First-order Raman amplification [24] or Second-order Raman amplification [25]. Considering that standard BOTDA schemes work at $\sim 1550$ $\mathrm{nm}$, First-order Raman amplification bases its principle on locating a pump at $1455 \mathrm{~nm}$ so that distributed amplification is produced at $1550 \mathrm{~nm}$ (see Fig.6(a)). For the case of Secondorder Raman amplification, the idea lays on locating a Firstorder pump at $1455 \mathrm{~nm}$ (one Stokes order difference with respect to the BOTDA signals) and a Second-order Raman pump at twice the Stokes difference (1365 nm roughly, see Fig.6(b)). The aim of this configuration is to avoid the effect of linear loss in the First-order pump and achieve a smooth compensation of the fiber losses. This allows achieving setups that almost work in virtual transparency, like the ones presented in references $[25,26]$. Both configurations lead to an enhancement of the measuring range without compromising the resolution.

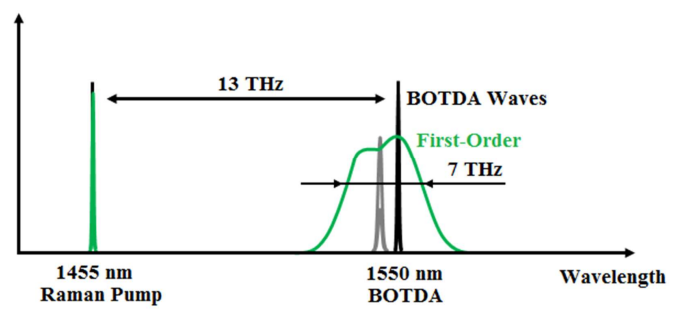

(a)

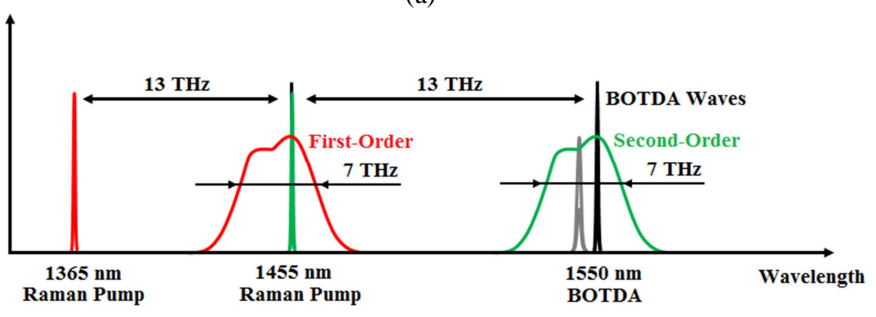

(b)

Fig. 6. Graphical representation of the First- (a) and Second-order (b) Raman amplification schemes in terms of signal placement in the spectrum.

As stated in Section II, typical BOTDA systems are limited in terms of power levels by the appearance of MI when referring to the pulsed pump wave $(\sim 100 \mathrm{~mW})$ and by SBS pump depletion for the continuous probe wave $(\sim 40 \mu \mathrm{W}$ for the SSP scheme and $\sim 5 \mathrm{~mW}$ for the DSP case). When distributed Raman amplification is introduced within the system, both pump and probe waves will be as well amplified, thus a proper trade-off must be encountered among the launched pump-probe waves and the Raman signals. In a 
previous work developed by Soto et al. [27], a detailed study of the effect of Raman amplification was performed in terms of pump and probe power levels (for first order schemes). In usual settings (for $>100 \mathrm{~km}$ setups), the Brillouin pump power has to be lowered to few tens of milliwatts (or even below) to avoid MI, and the probe wave has to be reduced to a few tens of microwatts to avoid non-local effects.

As any other optical amplification, Raman gain introduces ASE noise. Although this is a significant source of noise, it has not been the dominant source of noise in Raman-assisted BOTDA demonstrations up to now. The dominant source of noise in Raman-assisted BOTDA setups has been so far RIN transfer. The origin of the RIN problem in Raman assistance is that Raman assistance requires pumping the fiber with relatively high powers, so normally high-power emission is required at 1455 or $1365 \mathrm{~nm}$. To be able to provide considerable gain levels to long range systems, nowadays it is often necessary to resort to Raman fiber laser pumps which unfortunately introduce large amounts of RIN. These Raman amplifier sources, whether First- or Second-order, present considerable intensity fluctuations that lead to time-dependent variations on the produced Raman gain and end up being imprinted on the BOTDA probe $[28,29,30]$. The strength of the RIN transfer depends both on the gain (pumping level used) and the length of the fiber.

It is worth mentioning that besides from the cited First- and Second-order Raman amplification techniques, some long range BOTDA systems base the amplification system on the use of Ultra-long or Random Fiber Lasers (URFL) [31,32]. URFLs are developed by employing extremely long fibers and sufficiently high second order pumping. Rayleigh reflection in the fiber itself or suitable FBGs in the fiber ends provide the necessary feedback to achieve a laser in the sensing fiber itself. This laser turns out to be tuned to provide first-order Raman gain to the Brillouin signals. RIN transfer has been extensively analyzed and reported by Nuño et al. [34], showing distinctive RIN properties over other setups.

It should be clear that RIN noise is a technical noise, but not a fundamental limitation of the technique. The time-dependent variations present in the pump lasers are due to the own nature of the Raman fiber lasers employed to perform the distributed amplification. These pumps show a spectrum composed of many modes with a periodic spacing, that is related to the cavity round-trip time. The periodic mode spacing, which is normally in the hundreds of $\mathrm{kHz}$ or few $\mathrm{MHz}$ range for typical fiber lasers, leads to the appearance of some quasi-periodic intensity perturbations which may not be removed even after the usual trace averaging procedure. In particular, this occurs when some laser mode falls exactly on a multiple of the pump pulse repetition rate. To avoid this noise, several schemes have been put forward. The simplest is just controlling the pumping level up to the point of making the signal visible within the range of the detection setup [35]. It is also possible to employ low-RIN Raman pumps, e.g. Semiconductor Lasers (SL) [36]. In [37], a numerical de-noising technique was provided that bases its principle on the quasi-periodical nature of the noise source, which is recognizable in the FFT spectrum of the detected signal. In addition, it is also possible to shift the working frequency of the BOTDA to a frequency range in which the RIN transfer is smaller [38]. Recently, it has been shown that the RIN transfer problem can be completely solved using a dual-sideband probe scheme and balanced detection $[39,40]$.

Since the RIN problem is avoidable, to perform our analysis of the limits of the technique we will concentrate on the standard Raman amplification techniques [24,25] and their ultimate limits, given mainly by ASE noise and Rayleigh scattering.

First-order Raman amplification can be introduced in the BOTDA sensing scheme through three different configurations with respect to the pump wave; co-propagating, counter-propagating and bi-directionally to the pump pulse. When pumping in a co-propagating configuration to the pump wave, the maximum gain gets translated $10-15 \mathrm{~km}$ into the fiber. When introducing Raman amplification in a counterpropagating scheme, a better contrast at the far end of the fiber is achieved. Finally, a relatively good and effective cancellation of the fiber losses can be achieved by pumping bi-directionally in the fiber. Fig.7 shows a representation of the theoretical gain trace achieved in a First-order Ramanassisted configuration (peak pump power is $10 \mathrm{~mW}$, probe power is $50 \mu \mathrm{W}$ in dual sideband). As it is visible, over a 100 $\mathrm{km}$ fiber span the maximum gain can be as much as $1.4 \%$ while the minimum goes slightly below $0.2 \%$. For comparison, a grating-based Second-order setup is also shown, where the gain fluctuations are much smaller.

It is interesting to compare now the evolution of ASE noise in both cases. According to our models [24,25] and considering a conventional SMF-28 fiber, the ASE noise power obtained in the First-order configuration in the conditions of the previous sections (50 GHz optical filter) is $-39.85 \mathrm{dBm}$ in the First-order case and $-40.40 \mathrm{dBm}$ in the Second-order one. We are now interested in evaluating the worst-case SNR (i.e. the SNR in the position of worst contrast) using the same receiver used previously in the preamplifier case. The SNR in the worst case turns out to be roughly $27 \mathrm{~dB}$ in the First-order case and $30.6 \mathrm{~dB}$ in the Second-order scheme (in both cases, with 1024 averages), which is almost 9 and $12 \mathrm{~dB}$ better than the optimal SNR obtained in the pre-amplifier case and $15 \mathrm{~dB}$ and $18.6 \mathrm{~dB}$, respectively, better than the non-amplified case. This implies a range extension of $>37 \mathrm{~km}$ with the First-order technique and $>46 \mathrm{~km}$ in the Second-order case. Still, even though it can display remarkable sensing range extension, the approach is obviously limited and the complexity of the technique is considerable. 


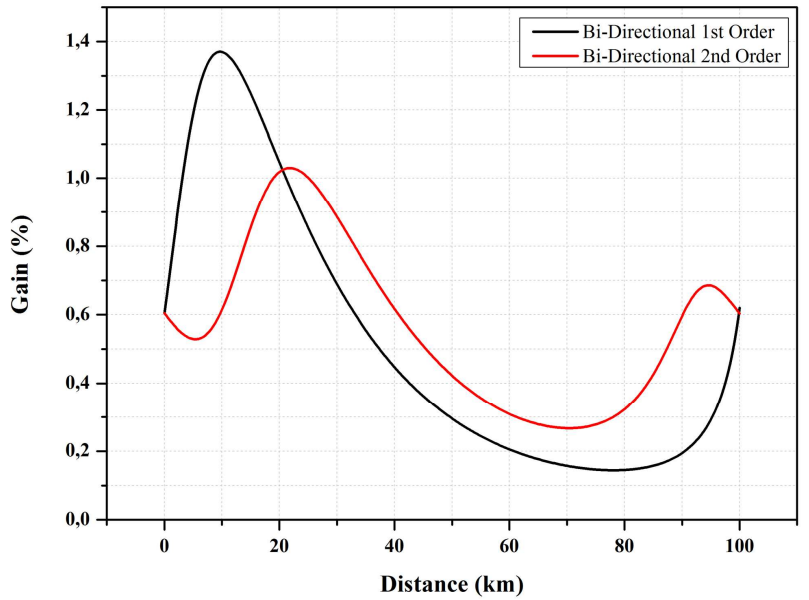

Fig. 7. Theoretical Brillouin Gain traces obtained as a function of distance in a Raman-assisted BOTDA using Bi-directional First- or Second-order pumping. Simulations have been done employing the equations contained in [24,25].

\section{DISCUSSION}

As we can see, none of the proposed three techniques (preamplification, pulse-coding and Raman amplification) provides an ultimate solution to extend the sensing range of BOTDA to fulfill the monitoring necessities of the market. So far, we have reported an increase of the detected noise in terms of ASE noise, a limitation of the employed code-length and a large amount of RIN transfer from high power laser sources.

Recently, Soto et al. have reported a way of rating the performance of the different available BOTDA techniques with a common Figure of Merit (FoM) [41]. Based on the already existing BOTDA bibliography, it is possible to evaluate the effect of the aforementioned techniques on the performance of Brillouin Time Domain sensors. In the following table (Table 1), we schematize the FoM of the different configurations already proven and their effect in terms of efficiency in the sensing range. The selected works are applied on similar range and resolution systems.

As we can see in Table 1, since the development of one of the first "long range" BOTDA in 1993 by Bao et al. [42], the technology has evolved enormously. In that case, the FoM ranged 2 principally due to the low resolution $(10 \mathrm{~m})$ of the system. With the introduction of pre-amplification [43], the range, as expected, was increased $\sim 20 \mathrm{~km}(\sim 8 \mathrm{~dB})$ and the resolution reduced until 2 meters. Obviously, the FoM is enhanced but the performance can still not be considered optimum for long-range applications. With the application of pulse coding [18] a performance improvement was obtained of almost a factor of 10 in comparison to the results of Bao et al. Raman amplification (First- [35] and Second-order [25]) implies a considerable breakthrough. The sensing range is increased until $100 \mathrm{~km}$ maintaining a 2 meter resolution. The figure of merit is improved $\sim 7$ times over the ones achieved with pre-amplification or coding. Unfortunately, the detrimental effect of RIN transfer from the Raman fiber sources makes necessary to increase the number of averages considerably, degrading the performance of the sensor in terms of measuring time. In case the RIN noise is removed, e.g. by applying the balanced detection technique [40], it is possible to significantly reduce the number of averages, thus upgrading the FoM up to 500 by using only Raman assistance. This is slightly beyond the expectation of FoM increase in the discussion of the present paper, but in reasonable agreement if we consider the evolution of components along the experiments. In any case, the range of $100 \mathrm{~km}$ with a single technique is not yet surpassed, but the combination of techniques seems to be the path to follow when trying to extend the sensing range beyond the $100 \mathrm{~km}$ barrier.

With the combination of coding with Raman amplification [44], it is possible to reduce considerably the average number until 508 for a sensing distance of $75 \mathrm{~km}$ with 2 meter resolution, which provides a FoM of 650 . When coding is combined with pre-amplification [45] it is possible to reach until $120 \mathrm{~km}$ with resolutions of few meters, but the number of averages is still rounding the two-five thousand scale, even though pulse codification is capable of reducing RIN transfer. The unification of all three techniques [46], is the one that provides a sensing range greater than $100 \mathrm{~km}$ with a 4 meter resolution and an averaging of 500. Obviously, the improvement is enormous, especially if compared with the first BOTDA systems.

If the amplification process is developed through the use of the previously described URFL the obtained performance enhances substantially when combined with a preamplification stage and pulse-coding [31,32]. For the case presented in [31] $142.2 \mathrm{~km}$ of sensing fiber are achieved for 5 meter resolution and 1024 equivalent averages, thus the FoM rapidly grows up to 26000 . For the case developed in [32] the Raman amplification is as well assisted by a low noise Firstorder Raman pump co-propagant to the probe wave so the possible RIN transfer to the detected probe wave is minimized. By such means, $154.4 \mathrm{~km}$ sensing range are obtained with 5 meter resolution and 4080 averages, almost doubling the previous FoM until 45000.

In all the proposed systems, the sensing range equals the total fiber length employed; therefore, if a linear measuring application is required, the maximum sensible distance is of "just" $\sim 60-75 \mathrm{~km}$, which sets back the performance of the systems if just pre-amplification or pulse coding is employed, and market requirements are not satisfied. That is why, recently, the combination of all three techniques was proposed (pre-amplification, pulse coding and First- and Second-order Raman) [47] to be able to break the $100 \mathrm{~km}$ limit but for linear sensing applications (> $200 \mathrm{~km}$ of total fiber). In that work, it was possible to reach $120 \mathrm{~km}$ of fiber ( $240 \mathrm{~km}$ of fiber loop) with 5 meter resolution and the equivalent of 2048 averages. Evidently, the FoM is quickly increased up to 300000 . This result was achieved principally due to an optimized Raman amplification configuration; the probe wave was amplified through a low noise First-order Raman pump so it is able to travel through the $120 \mathrm{~km}$ of lead fiber within a measurable power level, and the pump wave is amplified with an improved "seeded" Second-order Raman configuration $[48,49]$. 
TABLE I

COMPARISON AMONG THE DIFFERENT RANGE EXTENDER TECHNIQUES

\begin{tabular}{lcccc}
\hline \multicolumn{1}{c}{ Technology } & Range & Res. & Avg. & FoM \\
\hline Standard BOTDA [42] & $22 \mathrm{~km}$ & $10 \mathrm{~m}$ & 256 & 2 \\
Pre-Amplification [43] & $40 \mathrm{~km}$ & $2 \mathrm{~m}$ & 256 & 6.8 \\
Pulse Coding [18] & $50 \mathrm{~km}$ & $1 \mathrm{~m}$ & 2048 & 14 \\
$1^{\text {st }}$ Raman w/o RIN Cancellation [35] & $100 \mathrm{~km}$ & $2 \mathrm{~m}$ & 65536 & 110 \\
$2^{\text {nd }}$ Raman w/o RIN Cancellation [25] & $100 \mathrm{~km}$ & $2 \mathrm{~m}$ & 16000 & 290 \\
$1^{\text {st }}$ Raman with RIN Cancellation [40] & $100 \mathrm{~km}$ & $2 \mathrm{~m}$ & 1024 & 500 \\
Coding + $1^{\text {st }}$ Raman [44] & $75 \mathrm{~km}$ & $2.5 \mathrm{~m}$ & 508 & 650 \\
Pre-Amp. + Coding [45] & $120 \mathrm{~km}$ & $3 \mathrm{~m}$ & 2000 & 1800 \\
Pre-Amp. + Coding + $1^{\text {st }}$ Raman [46] & $122 \mathrm{~km}$ & $4 \mathrm{~m}$ & 508 & 13000 \\
Pre-Amp. + Coding + $2^{\text {nd }}$ URFL [32] & $142 \mathrm{~km}$ & $5 \mathrm{~m}$ & 1024 & 26000 \\
Pre-Amp. + Coding + $1^{\text {st }}-2^{\text {nd }}$ URFL [31] & $154.4 \mathrm{~km}$ & $5 \mathrm{~m}$ & 4080 & 45000 \\
Pre-Amp. + Coding + $1^{\text {st }}-2^{\text {nd }}$ Raman [47] & $120 \mathrm{~km}{ }^{(*)}$ & $5 \mathrm{~m}$ & 2040 & 300000 \\
\hline \hline (*): Lima Sensing Configution 240 km Fib Lowp & & & \\
\hline
\end{tabular}

(*): Linear Sensing Configuration - 240 km Fiber Loop.

\section{CONCLUSIONS}

In conclusion, it is evident that the combination of all the proposed techniques is the path to follow when extremely long distances are required. Obviously, the complexity of such systems is considerable too, but the performance enhancement is so palpable, that is completely worth it. The range achieved so far, provides an efficient sensing solution to most of the applications present up to now, but the possibility of succeeding on achieving $150 \mathrm{~km}$ of linear range will satisfy almost any sensing application. There are already some research groups and companies that are introducing balanced detection in their optimized long range BOTDA systems, which is probably the final improvement on the proposed schemes to break the $150 \mathrm{~km}$ barrier.

In this work, we have presented a measure of the limits of the available techniques to obtain extremely long sensing ranges on BOTDA systems. After a detailed revision of the available techniques (pre-amplification, pulse coding and Raman assistance) and the application of the FoM, it is concluded that the combination among techniques is the most effective way to break the limiting barrier of $100 \mathrm{~km}$. This has been demonstrated in recent literature results, which might be improved in the future.

\section{REFERENCES}

[1] T. Horiguchi, and M. Tateda, "Optical-fiber-attenuation investigation using stimulated Brillouin scattering between a pulse and a continuous wave," Opt. Lett., vol. 14, no. 8, pp. 408-410, 1989.

[2] T. Horiguchi, and M. Tateda, "BOTDA - Nondestructive Measurement of Single-Mode Optical Fiber Attenuation Characteristics Using Brillouin Interaction: Theory," IEEE J. Lightwave Technol., vol. 7, no. 8, pp. 1170-1176, 1989.

[3] G. P. Agrawal, Nonlinear Fiber Optics, $4^{\text {th }}$ ed. San Diego, CA: Academic, 2007, ch. 9.

[4] M. Niklés, "Fibre optic distributed scattering sensing system: Perspectives and challenges for high performance applications," in Third European Workshop on Optical Fiber Sensors, 66190D, Italy, 2007.

[5] M. Gonzalez-Herraez, A. Dominguez-Lopez, A. Lopez-Gil, H. F. Martins, S. Martin-Lopez, X. Angulo-Vinuesa and P. Corredera, "Longrange static and dynamic distributed sensing", presented at the IEEE Sensors 2014 Conference, 2014.
[6] L. Thévenaz and M. A. Soto, "Rating the performance of a Brillouin distributed fiber sensor", Proc. of SPIE, vol. 8421, pp. 8421A7, 2012.

[7] L. Thévenaz, S. Foaleng-Mafang, and J. Lin, "Effect of pulse depletion in a Brillouin optical time-domain analysis system," Opt. Express, vol. 21, no. 12, pp. 14017-14035, 2013.

[8] A. Minardo, R. Bernini, and L. Zeni, "A simple technique for reducing pump depletion in long-range distributed Brillouin fiber sensors," IEEE Sens. J., vol. 9, no. 6, pp. 633-634, 2009.

[9] S. Foaleng-Mafang and L. Thévenaz, "Impact of Raman scattering and modulation instability on the performances of Brillouin sensors," Proc. of SPIE, vol. 7753, pp. 77539V, 2011.

[10] A. Zornoza, A. Minardo, R. Bernini, A. Loayssa and L. Zeni, "Pulsing the Probe Wave to Reduce Nonlocal Effects in Brillouin Optical TimeDomain Analysis (BOTDA) Sensors," IEEE Sens. J., vol. 11, no. 4, pp. 1067-1068, 2011

[11] A. Minardo, R. Bernini, L. Zeni, L. Thevenaz, and F. Briffod, "A reconstruction technique for long-range stimulated Brillouin scattering distributed fiber-optic sensors: Experimental results," Meas. Sci. Technol., vol. 16, no. 4, pp. 900-908, 2005.

[12] Y. Dong, L. Chen and X. Bao, "Extending the Sensing Range of Brillouin Optical Time-Domain Analysis Combining FrequencyDivision Multiplexing and In-Line EDFAs," IEEE J. Lightwave Technol., vol. 30, no. 8, pp. 1161-1167, 2012.

[13] Y. Dong, L. Chen and X. Bao, "Time-division multiplexing-based BOTDA over $100 \mathrm{~km}$ sensing length," Opt. Lett., vol. 36, no. 2, pp. $277-$ $279,2011$.

[14] W. Li, X. Bao, Y. Li and L. Chen. "Differential pulse-width pair BOTDA for high spatial resolution sensing," Opt. Express, vol. 16, no. 26, pp. 21616-21625, 2008.

[15] K. De Souza and T. P. Newson, "Signal to noise and range enhancement of a Brillouin intensity based temperature sensor," Opt. Express, vol. 12, no. 12, pp. 2656-2661, 2004.

[16] E. Desurvire, Erbium-doped fiber amplifiers, John Wiley \& Sons, 1994, pp. 207-305.

[17] D. Lee, H. Yoon, N. Y. Kim, H. Lee and N. Park, "Analysis and experimental demonstration of Simplex coding technique for SNR enhancement of OTDR," presented at the Lightwave Technologies in Instrumentation and Measurement Conference, 2004.

[18] M. A. Soto, G. Bolognini, F. Di Pascuale and L. Thévenaz, "Simplexcoded BOTDA fiber sensor with $1 \mathrm{~m}$ spatial resolution over a $50 \mathrm{~km}$ range," Opt. Lett., vol. 35, no. 2, pp. 259-261, 2010.

[19] M. A. Soto, G. Bolognini, F. Di Pasquale and L. Thévenaz, "Long-range Brillouin optical time-domain analysis sensor employing pulse coding techniques," Meas. Sci. Technol., vol. 21, no. 9, pp.094024 (7pp), 2010.

[20] P. Corredera, M. L. Hernanz, M. González-Herráez and J. Campos, "Anomalous non-linear behavior of InGaAs photodiodes with overfilled illumination," Metrología, vol. 40, no. 1, pp. S150, 2003.

[21] M. A. Soto, G. Bolognini and F. Di Pasquale, "Analysis of pulse modulation format in coded BOTDA sensors," Opt. Express, vol. 18, no. 14, pp. 14878-14892, 2010.

[22] H. Liang, W. Li, N. Linze, L. Chen and X. Bao, "High-resolution DPPBOTDA over $50 \mathrm{~km}$ LEAF using return-to-zero coded pulses," Opt. Lett., vol. 35, no. 10, pp. 1503-1505, 2010 .

[23] R. W. Boyd, Nonlinear Optics, 3th ed. Academic Press, 2008

[24] F. Rodríguez-Barrios, S. Martín-López, A. Carrasco-Sanz, P. Corredera, J.D. Ania-Castañón, L. Thévenaz, and M. González-Herráez, "Distributed Brillouin Fiber Sensor Assisted by First-Order Raman Amplification," IEEE J. Lightwave Technol., vol. 28, no. 15, pp. 21622172, 2010.

[25] S. Martín-López, M. Alcón-Camas, F. Rodríguez, P. Corredera, J.D. Ania-Castañón, L. Thévenaz and M. González-Herráez, "Brillouin optical time-domain analysis assisted by second-order Raman amplification," Opt. Express, vol. 18, no. 18, pp. 18769-18778, 2010.

[26] M. Gonzalez-Herraez, S. Martin-Lopez, M. Alcon-Camas, P. Corredera, L. Thévenaz, J. D. Ania-Castañon, "Ultra-long range distributed fibre sensing using virtually transparent propagation," presented at the CLEO Europe, 2011.

[27] M. A. Soto, G. Bolognini and F. Di Pasquale, "Optimization of longrange BOTDA sensors with high resolution using first-order bidirectional Raman amplification," Opt. Express, vol. 19, no. 5, pp. 44444457, 2011.

[28] M. Alcon-Camas and J. D. Ania-Castañon, "RIN transfer in 2nd-order distributed amplification with ultralong fiber lasers," Opt. Express, vol. 18 , no. 23 , pp. $23569-23575,2010$. 
[29] C. R. S. Fludger, V. Handerek and R. J. Mears, "Pump to signal RIN transfer in Raman fiber amplifiers," IEEE J. Lightwave Technol., vol. 19, no. 8, pp. 1140-1148, 2001.

[30] B. Bristiel, S. Jiang, P. Gallion and E. Pincemin, "New model of noise figure and RIN transfer in fiber Raman amplifiers," IEEE Photon. Technol. Lett., vol. 18, no. 8, pp. 980-982, 2006.

[31] X.-H. Jia, Y.-J. Rao, C.-X. Yuan, J. Li, X.-D. Yan, Z.-N. Wang, W.-L. Zhang, H. Wu, Y.-Y. Zhu and F. Peng, "Hybrid distributed Raman amplification combining random fiber laser based 2nd-order and lownoise LD based 1st-order pumping," Opt. Express, vol. 21, no. 21, pp. 24611-24619, 2013.

[32] X.-H. Jia, Y.-J. Rao, Z.-N. Wang, W.-L. Zhang, C.-X. Yuan, X.-D. Yan, J. Li, H. Wu, Y.-Y. Zhu and F. Peng, "Distributed Raman amplification using ultra-long fiber laser with a ring cavity: characteristics and sensing application," Opt. Express, vol. 21, no. 18, pp. 21208-21217, 2013.

[33] S. K. Turitsyn, S. A. Babin, A. E. El-Taher, P. Harper, D. V. Churkin, S. I. Kablukov, J. D. Ania-Castañón, V. Karalekas and E. V. Podivilov, "Random distributed feedback fiber laser," Nat. Photonics, vol. 4, no. 4, pp. 231-235, 2010.

[34] J. Nuño, M. Alcon-Camas and J. D. Ania-Castañon, "RIN transfer in random distributed feedback fiber lasers," Opt. Express, vol. 20, no. 24, pp. 27376-27381, 2012.

[35] X. Angulo-Vinuesa, S. Martín-López, J. Nuno, P. Corredera, J. D. AniaCastanon, L. Thevenaz, and M. Gonzalez-Herraez, "Raman-assisted Brillouin distributed temperature sensor over $100 \mathrm{~km}$ featuring $2 \mathrm{~m}$ resolution and $1.2^{\circ} \mathrm{C}$ uncertainty," IEEE J. Lightwave Technol., vol. 30, no. 8, pp. 1060-1065, 2012.

[36] M. A. Soto, G. Bolognini and F. Di Pasquale, "Optimization of longrange BOTDA sensors with high resolution using first-order bidirectional Raman amplification," Opt. Express, vol. 19, no. 5, pp. 4444 4457, 2011.

[37] X. Angulo-Vinuesa, S. Martin-Lopez, P. Corredera, and M. GonzalezHerraez, "Raman-assisted Brillouin optical time-domain analysis with sub-meter resolution over 100 km," Opt. Express, vol. 20, no. 11, pp. 12147-12154, 2012

[38] X. Angulo-Vinuesa, D. Bacquet, S. Martin-Lopez, P. Corredera, P. Szriftgiser and M. Gonzalez-Herraez, "Relative Intensity Noise transfer reduction in Raman-assisted BOTDA systems," IEEE Photon. Technol. Lett., vol. 26, no. 3, pp. 271-274, 2014.

[39] A. Dominguez-Lopez, A. Lopez-Gil, S. Martin-Lopez and M. GonzalezHerraez, "Signal to noise ratio improvement in BOTDA using balanced detection", IEEE Photon. Technol. Lett., vol. 26, no. 4, pp. 338-341, 2014.

[40] A. Dominguez-Lopez, A. Lopez-Gil, S. Martin-Lopez and M. GonzalezHerraez, "Strong cancellation of RIN noise in a Raman-assisted BOTDA using balanced detection", IEEE Photon. Technol. Lett., vol. 26, no. 18, pp. 1817-1820, 2014.

[41] M. A. Soto and L. Thévenaz, "Modeling and evaluating the performance of Brillouin distributed optical fiber sensors," Opt. Express, vol. 21, no. 25, pp.31347-31366, 2013.

[42] X. Bao, D. J. Webb, and D. A. Jackson, "22-km distributed temperature sensor using Brillouin gain in an optical fiber," Opt. Lett., vol. 18, no. 7, pp. 552-554, 1993.

[43] S. Diaz, S. Foaleng Mafang, M. Lopez-Amo and L. Thevenaz, "A highperformance optical time-domain Brillouin distributed fiber sensor," IEEE Sens. J., vol. 8, no. 7, pp. 1268-1272, 2008.

[44] X.-H. Jia, Y.-J. Rao, K. Deng, Z.-X. Yang, L. Chang, C. Zhang and Z.L. Ran, "Experimental Demonstration on 2.5-m Spatial Resolution and 1 ${ }^{\circ} \mathrm{C}$ Temperature Uncertainty Over Long-Distance BOTDA With Combined Raman Amplification and Optical Pulse Coding," IEEE Photon. Technol. Lett., vol. 23, no. 7, pp. 435-437, 2011.

[45] M. A. Soto, G. Bolognini and F. Di Pasquale, "Long-range simplexcoded BOTDA sensor over $120 \mathrm{~km}$ distance employing optical preamplification," Opt. Lett., vol. 36, no. 2, pp. 232-234, 2011.

[46] X.-H. Jia, Y.-J. Rao, Z.-N. Wang, W.-L. Zhang, Y. Jiang, J.-M. Zhu and Z.-X. Yang, "Towards fully distributed amplification and highperformance long-range distributed sensing based on random fiber laser," Proc. of SPIE, vol. 8421, pp. 842127, 2012.

[47] M. A. Soto, X. Angulo-Vinuesa, S. Martin-Lopez, S.-H. Chin, J. D. Ania-Castañon, P. Corredera, E. Rochat, M. Gonzalez-Herraez, and L. Thévenaz, "Extending the Real Remoteness of Long-Range Brillouin Optical Time-Domain Fiber Analyzers," IEEE J. Lightwave Technol., vol. 32 , no. 1, pp. 152-162, 2014.

[48] S. Faralli, G. Bolognini, G. Sacchi, S. Sugliani and F. Di Pasquale, "Bidirectional higher order cascaded Raman amplification benefits for
10-Gb/s WDM unrepeated transmission systems," IEEE J. Lightwave Technol., vol. 23, no. 8, pp. 2427-2433, 2005.

[49] S. Faralli, G. Bolognini, M. A. Andrade, and F. Di Pasquale, "Unrepeated WDM transmission systems based on advanced first-order and higher order Raman-copumping technologies," IEEE J. Lightwave Technol., vol. 25, no. 11, pp. 3519-3527, 2007.

Xabier Angulo-Vinuesa was born in Bilbao, Spain, in 1982. He received the Electronic Engineering degree from the University of the Basque Country, Leioa, Spain, in 2009, and the Ph.D. degree in Advanced Electronics from the Univeristy of Alcalá, Alcalá de Henares, Spain, in 2014.

His thesis dissertation was based on the development of ultra-long range BOTDA systems. During his Ph.D. he has visited the laboratories from the Group for Fiber Optics at the Swiss Federal Institute of Technology of Lausanne (EPFL), Lausanne Switzerland, and the Laser, Atoms and Molecule's Physics Laboratory (PhLAM) at the University of Lille 1, Villeneuve d'Asq, France. Since July 2014 he is a Postdoctoral Research at the Photonics Engineering Group of the University of Alcalá. His main research interests include optical fiber sensing, optical amplification systems and nonlinearities in optical fibers.

Alejandro Dominguez-Lopez was born in Granada, Spain, in 1987. He received the Telecommunication engineering degree from the University of Granada, Granada, Spain, in 2012 and the Master degree in advanced electronics systems from the University of Alcalá, Madrid, Spain, in 2014. Since 2013, he has been working towards the Ph.D. degree in the Photonics Engineering Group, University of Alcala, Madrid, Spain.

His current research interests include nonlinear fiber optics and fiber optic sensors.

Alexia Lopez-Gil received the Telecommunication engineering degree from the Polytechnic University of Madrid, Madrid, Spain, in 2012 and the Master degree in advanced electronics systems from the University of Alcalá, Madrid, Spain, in 2014. Since 2013, she has been working towards the Ph.D. degree in the Photonics Engineering Group, University of Alcala, Madrid, Spain.

Her current research interests include nonlinear fiber optics and fiber optic sensors.

Juan Diego Ania-Castañón was born in Oviedo, Spain, in 1973. He received a MSc. degree in Astrophysics from Universidad Complutense de Madrid, Madrid, Spain, in 1996, and a PhD. degree in Theoretical Physics from Universidad de Oviedo and Instituto de Estructura de la Materia, Consejo Superior de Investigaciones Científicas (CSIC), Madrid, Spain, in 2000.

In 2001, he joined the Photonics Research Group, Aston University, Birmingham, U.K.. In 2007, he took a permanent position at the Nonlinear Dynamics and Fiber Optics Research Group, Instituto de Óptica, CSIC. He is the author or coauthor of about 140 papers in international refereed journals and conference proceedings. His main research interests include the study of nonlinear dynamics in optical systems and the exploitation of nonlinear effects in optical fibers. 
Sonia Martin-Lopez received the $\mathrm{Ph} . \mathrm{D}$ degree from the Universidad Complutense de Madrid in May 2006.

The topic of her doctoral dissertation was on experimental and theoretical understanding of continuous-wave pumped supercontinuum generation in optical fibers. She had a stay in the Nanophotonics and Metrology Laboratory, Ecole Polytechnique Federale de Lausanne, Switzerland. She has been engaged as post-doctoral researcher in the Applied Physics Institute and in the Optics Institute at the Spanish Council for Research. She is currently engaged as postdoctoral Ramon y Cajal researcher in the Electronic Department of Alcala University of Madrid. She is author or co-author of over 100 papers in international refereed journals and conference contribution. Her current research interests include nonlinear fiber optics and optical fiber sensors.

Miguel Gonzalez-Herraez received the M.Eng. and D.Eng. degrees from the Polytechnic University of Madrid, Madrid, Spain, in 2000 and 2004, respectively.

While working toward the D.Eng. degree, he worked first as a Research Assistant and then a Postdoctoral Fellow in the Applied Physics Institute at the Spanish Council for Research, Madrid, Spain, and had several long stays in the Nanophotonics and Metrology Laboratory, Ecole Polytechnique Federale de Lausanne, Switzerland. In October 2004, he was appointed Assistant Professor in the Department of Electronics, University of Alcalá, Madrid, Spain, where he was promoted to Associate Professor in June 2006. He is the author or coauthor of more than 160 papers in international refereed journals and conference contributions and has given several invited talks at international conferences. His research interests include the wide field of nonlinear interactions in optical fibers.

Dr. González-Herráez has received several important recognitions to his research career, including the European Research Council Starting Grant and the "Agustin de Betancourt" prize of the Spanish Royal Academy of Engineering. 